\title{
Magnetohydrostatic (MHS) atmospheres ${ }^{\star}$
}

\author{
L. J. November ${ }^{\star \star}$
}

The Light Physics, PO Box 217, La Luz, NM 88337-0217, USA

Received 29 January 2003 / Accepted 18 December 2003

\begin{abstract}
We show that the atmospheric and magnetic height variations are coupled in general MHS equilibria with gravity when isolated thin non-force-free flux tubes are present. In gas-dominated environments, as in stellar photospheres, flux tubes must expand rapidly with height to maintain pressure balance with the cool surroundings. But in magnetically dominated environments, as in stellar coronae, the large-scale background magnetic field determines the average spreading of embedded flux tubes, and rigidly held flux tubes require a specific surrounding atmosphere with a unique temperature profile for equilibrium. The solar static equilibrium atmosphere exhibits correct transition-region properties and the accepted base coronal temperature for the sun's main magnetic spherical harmonic. Steady flows contribute to the overall pressure, so equilibria with accelerated wind outflows are possible as well. Flux tubes reflect a mathematical degeneracy in the form of non-force-free fields, which leads to coupling in general equilibrium conditions. The equilibrium state characterizes the system average in usual circumstances and dynamics tend to maintain the MHS atmosphere. Outflows are produced everywhere external to rigidly held flux tubes that refill a depleted or cool atmosphere to the equilibrium gas profile, heating the gas compressively.
\end{abstract}

Key words. magnetohydrodynamics (MHD) - Sun: atmosphere - Sun: corona - Sun: transition region - stars: coronae stars: winds, outflows

\section{Introduction}

In our observation of solar "threads" (November \& Koutchmy 1996, hereafter NK96) we puzzled over whether coronal flux tubes should expand with height to maintain pressure balance with the ambient gas-pressure decrease or follow the lines of the external magnetic field. After all, if the corona is independently heated, the gas and magnetic height variations should be generally different. The many observations of EUV and X-ray loops as well as the purely density-sensitive observations of white-light > 15 Mm "voids" (MacQueen et al. 1974), >2 Mm threads, and scintillation $>1 \mathrm{~km}$ filamentary microstructure (Coles \& Harmon 1978; Woo et al. 1995) all suggest that the large-scale solar coronal magnetic field is interspersed with flux tubes with a great range of sizes. Figure 1 shows dark and bright threads in a square region about a solar radius on a side over the west limb taken with the Canada-France-Hawaii Telescope (CFHT) at the unique total-eclipse opportunity on Mauna Kea on July 11, 1991. The dark and bright threads, which were essentially unchanged over the 4-min eclipse duration, appear to be aligned and organized in arched and radial surfaces, probably current sheets, overlapping in their projection onto the plane of the sky in the line-of-sight view.

We know that isolated thin flux tubes must expand with height in balance with the ambient gas-pressure decrease. The flux-tube gas pressure difference, external minus internal,

* All Appendices are only available in electronic form at http://www .edpsciences.org

$\star \star$ e-mail: laluzphys@yahoo.com

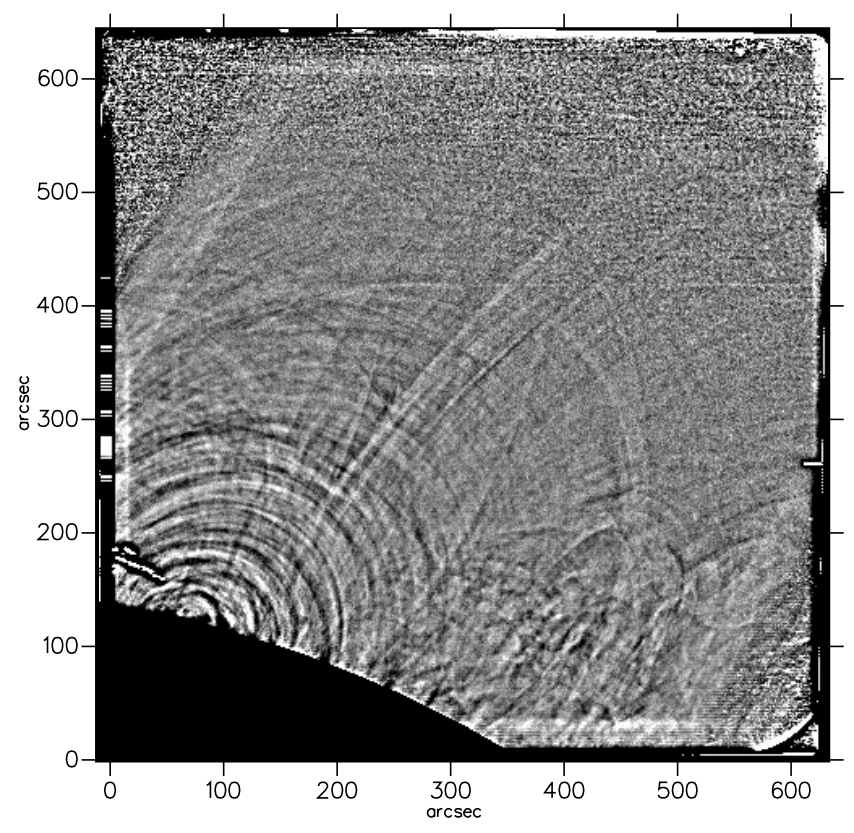

Fig. 1. Unsharp-masked time-averaged white-light eclipse image processed by subtracting a 6 arcsec $=4300 \mathrm{~km}$ Gaussian convolution shown with contrast range $\Delta \ln I=0.8 \%$. Dark and bright threads are probably flux tubes in current sheets.

is related to the magnetic pressure difference by the static equilibrium relation

$p_{\mathrm{e}}-p_{\mathrm{i}}=\frac{1}{8 \pi}\left(\boldsymbol{B}_{\mathrm{i}}^{2}-\boldsymbol{B}_{\mathrm{e}}^{2}\right)$. 
The Wilson depression in sunspots shows that at least largescale photospheric flux tubes are nearly evacuated. Evacuated flux tubes exhibit the maximum magnetic flux concentration and field strength possible. In evacuated flux tubes $p_{\mathrm{i}}=0$ and the photospheric external field $\boldsymbol{B}_{\mathrm{e}}=0$, so the internal field strength must follow the ambient gas-pressure decrease $\boldsymbol{B}_{\mathrm{i}}(r)^{2}=8 \pi p_{\mathrm{e}}(r)$, giving flux tubes that expand with height to conserve their total internal magnetic flux. Since the scale height for $p_{\mathrm{e}}(r)$ is small in the relatively cool photosphere and chromosphere, a dramatic flux-tube expansion occurs, which is the accepted explanation for the solar "magnetic canopy" (Giovanelli 1982). Both voids and a range of sizes of dark threads were also found to have contrasts consistent with what is expected for fully evacuated cylindrical flux tubes (MacQueen et al. 1983; NK96). In the corona, where the external magnetic field is not zero, the magnetic flux inside evacuated flux tubes must decrease with height to follow the total external ambient gas plus magnetic pressures, or $\boldsymbol{B}_{\mathrm{i}}(r)^{2}=8 \pi p_{\mathrm{e}}(r)+\boldsymbol{B}_{\mathrm{e}}(r)^{2}$. This gives a small flux-tube expansion with height in the large gas-pressure scale height of the hot corona. A slight flux-tube expansion with height seems evident in some of the threads in Fig. 1.

We can argue too that the radial expansion of isolated thin flux tubes must conform to the external magnetic field. Parker (1972) shows that

$$
\left(\boldsymbol{B}_{\mathrm{e}} \cdot \boldsymbol{\nabla}\right) \boldsymbol{B}_{\mathrm{i}}=0
$$

in MHS equilibria without gravity, where $\boldsymbol{B}_{\mathrm{e}}$ represents a uniform background magnetic field, and the field in flux tubes $\boldsymbol{B}_{\mathrm{i}}$ contains all of the spatial variations. The theorem is locally applicable everywhere in perturbed MHS equilibria with gravity, i.e. Eq. (2) with $\boldsymbol{B}_{\mathrm{e}}$ allowed to vary slowly spatially, as we discuss in Sect. 2. Thus thin flux tubes in a gravitational field must spread to follow the lines of the relatively uniform background magnetic field; the radial functional variations must be directly related, that is $\left|\boldsymbol{B}_{\mathrm{i}}(r)\right| \propto\left|\boldsymbol{B}_{\mathrm{e}}(r)\right|$ along flux tubes.

Therefore both ways of looking at the problem are essentially correct. The coronal flux-tube field must comply with the external field $\boldsymbol{B}_{\mathrm{i}}(r)^{2} \propto \boldsymbol{B}_{\mathrm{e}}(r)^{2}$, and the radial variation of the external gas pressure outside isolated thin evacuated coronal flux tubes is proportional to the magnetic pressure difference $p_{\mathrm{e}}(r) \propto \boldsymbol{B}_{\mathrm{i}}(r)^{2}-\boldsymbol{B}_{\mathrm{e}}(r)^{2}$. Hence, the external gas and magnetic pressures must be coupled with $p_{\mathrm{e}}(r) \propto \boldsymbol{B}_{\mathrm{e}}(r)^{2}$ outside and along isolated thin evacuated flux tubes. The background magnetic field is dominant in lower stellar coronae and must determine the expansion of embedded flux tubes. The expansion of evacuated flux tubes then defines the radial variation of the ambient gas pressure for equilibrium, the gas pressure being horizontally stratified in the external force-free or potential field. More generally, flux tubes of arbitrary base pressure but in thermal balance with the local ambient atmosphere satisfy $p_{\mathrm{i}}(r) \propto p_{\mathrm{e}}(r)$, giving the same external atmosphere $p_{\mathrm{e}}(r) \propto \boldsymbol{B}_{\mathrm{e}}(r)^{2}$. Thus an atmosphere containing isolated thin non-force-free flux tubes in different forms of local balance must exhibit a temperature profile defined by the dominant background magnetic field for a perfect gas in hydrostatic equilibrium.
The properties of flux tubes reflect a natural degeneracy in the form of non-force-free magnetic fields, and imply the existence of an underlying mathematical relationship between an equilibrium atmosphere and large-scale magnetic field. In Sect. 2, we show that the height variations of the gas pressure and magnetic field amplitude are directly coupled in the mostsignificant-order terms of the gravitational perturbation in general non-force-free MHS equilibria, with smaller field divergence and curl effects. In low- $\beta$ systems, with strong magnetic fields, the external gas pressure corresponds to a small difference $p_{\mathrm{e}}(r)=\left(\boldsymbol{B}_{\mathrm{i}}(r)^{2}-\boldsymbol{B}_{\mathrm{e}}(r)^{2}\right) /(8 \pi)$, which allows large pressure deviations for a given relative flexibility of the large-scale magnetic field around individual flux tubes. Magnetic fields can exhibit and persist in significant deviations from equilibrium, however the coupling $p_{\mathrm{e}}(r) \propto \boldsymbol{B}_{\mathrm{e}}(r)^{2}$ describes the general equilibrium condition for arbitrary $\beta$.

Historical MHS equilibrium solutions with gravity have also suggested that a general relationship exists between the large magnetic and gas-pressure scales. Dungey (1953) derived plane-parallel MHS equilibria for a magnetic field with its degenerate axis directed perpendicular to gravity (see also: Low 1975; Cheng \& Choe 1998). His solutions are represented in a modified Grad-Shafranov (GS) equation that retains the gaspressure radial scale-height variation as a separable factor in the pressure term. Dungey showed that when the magnetic field is also zero in its degenerate axis, the separable factor can be absorbed into the coordinates, giving the classical GS equation in a remapped coordinate system. The new GS coordinates are defined by the gas-pressure scale height, meaning that magnetic fields in a prespecified atmosphere must be spatially distorted. Horizontal flux tubes exhibit substantial radial deformation in a solar corona of given constant temperature (Zweibel $\&$ Hundhausen 1982; Low 1992). Depending upon their size compared to the gas-pressure scale height, flux tubes are either compressed or expanded in the gravitational direction, and approximately undistorted only in a specific atmosphere.

In Sect. 3, we examine equilibrium radial profiles of temperature and flux-tube diameter using an approximate solution to the equilibrium equations. The profiles reproduce the salient features of the solar atmosphere: the rapid expansion of flux tubes in the photosphere where the atmospheric gas pressure is dominant, a transition region of reasonable thickness located just above the height where the gas and magnetic energy densities are equal, and a corona of correct base temperature for the sun's main magnetic spherical harmonic. Steady flows do not alter the coupling, but may produce a Bernoulli pressure, which modifies the gas pressure, and leads to possible accelerated steady wind solutions.

Although the dynamics of the system can be quite complicated, the equilibrium state characterizes the average in many types of MHD systems, e.g. "quasi-steady" (Low 1980). Hydrodynamics in such systems tend to maintain the MHS atmosphere, as we discuss in Sect. 4. The possible importance of MHS atmospheres for other problems in physics and astrophysics beyond the formation of solar and stellar coronae and in more marginal non-quasi-steady physical conditions needs to be considered, but discussion lies beyond the scope of this paper. 


\section{MHS equilibria with gravity}

The equations for static equilibrium in a gravitational field are the MHS equation and Gauss' Law

$$
\begin{aligned}
& \boldsymbol{\nabla} p+\frac{p}{h} \nabla r=\frac{1}{4 \pi}(\boldsymbol{\nabla} \times \boldsymbol{B}) \times \boldsymbol{B}, \\
& \boldsymbol{\nabla} \cdot \boldsymbol{B}=0 .
\end{aligned}
$$

The static contribution to the pressure scale height $h$ is directly related to the temperature for a perfect gas, $h \equiv p /(\rho g(r))=$ $k T /(\bar{m} g(r))$. The variables have their usual meanings: $\boldsymbol{B}$ is the magnetic field vector in Gaussian units, $p$ the gas pressure, $\rho$ the density, $T$ the temperature, $\bar{m}$ the mean particle mass, $k$ Boltzman's constant, $c$ the speed of light (used below); $g(r)=$ $G M_{\mathrm{s}} / r^{2}$ is the gravitational acceleration, with $G$ the universal gravitational constant and $M_{\mathrm{s}}$ the stellar mass. The coordinate $r$ measures the distance from the system or stellar center, and the formal notation $\boldsymbol{\nabla} r$ is adopted for algebraic convenience to denote the radially directed unit vector.

The set of four vector-element Eqs. (3) and (4) contain five unknowns, $p, h$, and the three vector elements of $\boldsymbol{B}$. However, the solution space for $\boldsymbol{B}$ is more restrictive than would be obtained with linear relations, as we discuss in this section and elaborate in Appendix A.

The gravitational term in Eq. (3) compared to the pressure gradient is of order $d / h$, where $d$ is a characteristic flux-tube thickness; for the main power in threads in the solar corona $d / h<10^{-3}$. In the vicinity of non-force-free fields gas-pressure changes must be mainly magnetically determined, and only far away can the gravitational gradient be significant. Where the Lorentz term is negligible, the gas pressure is hydrostatic and defined by a single radial temperature profile. Since the gravitational term is small in non-force-free conditions, the classical equilibrium solutions without gravity described in Appendices A and B are applicable, and Eqs. (1) and (2) are valid in every small volume, but with the allowance that quantities may vary slowly spatially. Thus we obtain coupled atmospheric and magnetic height variations, at least if certain types of flux tubes are present as described in the Introduction.

Conditions along field lines are represented by a parallelfield equation

$\boldsymbol{B} \cdot\left(\boldsymbol{\nabla} p+\frac{p}{h} \boldsymbol{\nabla} r\right)=0$,

which is the projected component of the MHS Eq. (3) along $\boldsymbol{B}$. The relation says that gas pressure changes along field lines must be hydrostatic, which can be seen by introducing the integrating factor $\phi$ into the gas pressure

$p=\phi \hat{p}$,

for an unrestricted function $\hat{p}$. Upon substitution

$\boldsymbol{B} \cdot\left(\phi \boldsymbol{\nabla} \hat{p}+\hat{p}\left(\boldsymbol{\nabla} \phi+\frac{\phi}{h} \boldsymbol{\nabla} r\right)\right)=0$,

so taking for the integrating factor

$\boldsymbol{\nabla} \ln \phi=-\frac{1}{h} \nabla r$ we obtain $\boldsymbol{B} \cdot \boldsymbol{\nabla} \hat{p}=0$, or $\hat{p}$ constant along field lines. The only solutions to Eq. (8) are the 1D $\phi=\phi(r)$ and $h=h(r)$ or $T=T(r)$, represented in the hydrostatic relation

$\frac{\partial \ln \phi(r)}{\partial r}=-\frac{1}{h(r)}$

which can be integrated to give

$\phi(r)=\exp \left(-\int_{r_{\mathrm{s}}}^{r} \frac{\mathrm{d} r}{h(r)}\right)$,

where $r_{\mathrm{s}}$ denotes an arbitrary base height for the radial variations. Thus pressure variations along arbitrarily directed field lines follow the 1D hydrostatic scale-height function $\phi(r)$ from a constant base pressure $\hat{p} ; 3 \mathrm{D}$ effects are possible because the base pressure $\hat{p}$ and the scale-height function $\phi(r)$ or temperature profile $T(r)$ can vary from field line to field line. One scale-height function $\phi(r)$ with temperature profile $T(r)$ applies throughout force-free regions and in isolated non-forcefree flux tubes in thermal balance with their local surroundings.

Substituting Eq. (6) into the MHS Eq. (3) and using Eq. (8) gives the cross-field equation

$\phi(r) \boldsymbol{\nabla} \hat{p}=\frac{1}{4 \pi}(\boldsymbol{\nabla} \times \boldsymbol{B}) \times \boldsymbol{B}$,

for $\hat{p}$ allowed to vary from field line to field line. We adopt the single scale-height function $\phi(r)$, assuming for our argument that one temperature profile applies throughout. At a base height $r_{\mathrm{s}}, \phi\left(r_{\mathrm{s}}\right)=1$, and the cross-field Eq. (11) resembles the classical MHS Eq. (A.1) without gravity. Since the base height $r_{\mathrm{s}}$ is arbitrary, it is again evident that the classical equilibrium solutions must be applicable in every small volume.

Since the base pressure $\hat{p}$ is constant along field lines, the variation in the scale-height function $\phi(r)$ on the left side of Eq. (11) must be reproduced on the right side in the Lorentz term, and so in $\boldsymbol{B}^{2}$ too. Thus $\boldsymbol{B}$ must vary like $\phi(r)^{1 / 2}$ along field lines in non-force-free fields within $d / h$, which is the order of the derivatives of the scale-height function. Formally we can absorb $\phi(r)$ into the magnetic field on the right side by decomposing $\boldsymbol{B}$ into the general product

$\boldsymbol{B}=\phi_{B}(r) \hat{\boldsymbol{B}}=\phi_{B 2}(r)^{1 / 2} \hat{\boldsymbol{B}}=\phi(r)^{1 / 2} \zeta(r) \hat{\boldsymbol{B}}$.

A 1D magnetic scale-height function $\phi_{B}(r)$ is sufficient to cancel $\phi(r)$; we introduce the residual radial multiplier $\zeta(r)$ to allow differing scale-height functions, leaving $\hat{\boldsymbol{B}}$ an unrestricted vector function. Substituting back into Eqs. (11) and (4) eliminates $\phi(r)$ and gives

$$
\begin{aligned}
& \boldsymbol{\nabla} \hat{p}=\frac{1}{4 \pi}\left(\left(\boldsymbol{\nabla}-\frac{\boldsymbol{\nabla} r}{2 h(r)}\right) \times \zeta(r) \hat{\boldsymbol{B}}\right) \times \zeta(r) \hat{\boldsymbol{B}}, \\
& \boldsymbol{\nabla} \cdot \zeta(r) \hat{\boldsymbol{B}}=\frac{\boldsymbol{\nabla} r}{2 h(r)} \cdot \zeta(r) \hat{\boldsymbol{B}} .
\end{aligned}
$$

The equations can be solved by substituting perturbative expansions in powers of the small quantity $d / h(r), \hat{p}=$ $\sum_{n} \hat{p}_{n}(d / h(r))^{n}$ and $\hat{\boldsymbol{B}}=\sum_{n} \hat{\boldsymbol{B}}_{n}(d / h(r))^{n}$ for $n \geq 0$ with $d$ a constant characteristic flux-tube thickness. The expansions 
separate according to powers of $(d / h(r))^{n}$, giving the mostsignificant order-zero equations

$$
\begin{aligned}
& \boldsymbol{\nabla} \hat{p}_{0}=\frac{1}{4 \pi}\left(\boldsymbol{\nabla} \times \zeta(r) \hat{\boldsymbol{B}}_{0}\right) \times \zeta(r) \hat{\boldsymbol{B}}_{0}, \\
& \boldsymbol{\nabla} \cdot \zeta(r) \hat{\boldsymbol{B}}_{0}=0 .
\end{aligned}
$$

Equations (15) and (16) in $\hat{p}_{0}$ and $\zeta(r) \hat{\boldsymbol{B}}_{0}$ are equivalent to the classical plane-parallel MHS equations without gravity, which are discussed in Appendix A. The solutions are the classical equilibria, which exhibit a degenerate direction $z$ in the local coordinate system $(x, y, z)$ taken to be arbitrarily oriented with respect to the radial $r$. There is no loss in generality in just taking $\zeta(r)=1$ and $\phi_{B 2}(r)=\phi(r)$ in Eq. (12) leaving $\hat{\boldsymbol{B}}_{0}$ the unperturbed solution from Eq. (A.5) and constant in $z$. If $z \not \perp r$ the solutions for $\boldsymbol{B}$ exhibit a variation along the field direction that goes like $\phi(r)^{1 / 2}$. In the case considered by Dungey (1953) with $z \perp r$, distortion effects are introduced perpendicular to the main magnetic field direction as described in the Introduction. Thus $h_{B}(r)=2 h(r)$ at every $r$, where the magnetic scale height is defined in the usual way, $h_{B}(r)=-\left[\partial \ln \phi_{B}(r) / \partial r\right]^{-1}$. The scale heights are coupled to most-significant order in the perturbing gravitational field in all non-force-free MHS equilibria containing thin flux tubes in thermal balance with their local surroundings.

Additional perturbative equations for the base functions $\hat{p}$ and $\hat{\boldsymbol{B}}$ originate with the rescaling of the magnetic field vector in $r$ as discussed in Appendix C. The rescaling leads to the small term $\nabla r /(2 h(r))$ in both Eqs. (13) and (14). The term appears on the right side of Eq. (14) and is needed since a slow radial decrease in $|\boldsymbol{B}|$ cannot occur without a divergence of field lines; it represents a compensating creation of flux in $\hat{\boldsymbol{B}}(r)$ with $r$. The term is subtracted from the curl in the Lorentz force in Eq. (13) and corresponds to a small residual pseudo-force directed between $\hat{\boldsymbol{B}}$ and the outward radial $\boldsymbol{\nabla} r$; it represents a progressive left-handed twist of field lines at the rate of $1 \mathrm{rad}$ in every magnetic scale height $2 h(r)$. In MHS equilibria with gravity, the amplitude, the divergence, and the curl of $\boldsymbol{B}$ all vary on the large scale $2 h(r)$.

In principle anyway, a single thin flux tube somewhere in the atmosphere, evacuated or in thermal balance with its surroundings, fixes the equilibrium gas pressure for the entire atmosphere, but all non-force-free fields must be consistent to satisfy mutually the horizontal pressure boundary condition. In reality of course, magnetic fields may exhibit local deviations, and the equilibrium state might only be reflected in the average in an atmosphere containing many flux tubes.

A flux tube in thermal balance satisfies the condition $p_{\mathrm{e}}(r) \propto \boldsymbol{B}_{\mathrm{e}}(r)^{2}$ whether of increased or decreased field strength compared to the local background magnetic field. A flux tube with increased field strength $\left|\boldsymbol{B}_{\mathrm{i}}(r)\right|>\left|\boldsymbol{B}_{\mathrm{e}}(r)\right|$, must exhibit a decreased internal gas pressure in Eq. (1), $p_{\mathrm{i}}(r)<p_{\mathrm{e}}(r)$ consistent with the extreme dark-thread evacuated case of $p_{\mathrm{i}}(r)=0$, which requires the minimum surrounding atmosphere for a given magnetic pressure difference. Flux tubes with less field strength than the external magnetic field $\left|\boldsymbol{B}_{\mathrm{i}}(r)\right|<\left|\boldsymbol{B}_{\mathrm{e}}(r)\right|$, must have an increased gas pressure $p_{\mathrm{i}}(r)>p_{\mathrm{e}}(r)$, consistent with bright threads.
Threads appear to be isolated thin flux tubes aligned with the background magnetic field and embedded in current sheets in a bimodal amplitude distribution, consistent with the natural equilibrium form discussed in Appendix B. The temperature of bright threads was indeed found to be the same as the external surroundings within observational uncertainties (NK96). The solar EUV coronal temperature for unresolved quiet and active regions is relatively uniform, $1.5-2.1 \times 10^{6} \mathrm{~K}$ (Withbroe 1975), and a narrow temperature range around $2 \times 10^{6} \mathrm{~K}$ is found for a broad range of sizes and densities of X-ray loops for all but prominence flare loops (Davis et al. 1975; Vaiana et al. 1976; Rosner et al. 1978). Large-area coronal temperatures derived from the solar white-light scale height and forbidden line ratios give temperatures somewhat lower than the EUV and X-ray temperatures, in the range of $1-2 \times 10^{6} \mathrm{~K}$ at the solar radial height $r=1.15 r_{\odot}$ (Guhathakurta et al. 1992).

A slightly heated (or cooled) flux tube exhibits an increased (or decreased) internal pressure $p_{\mathrm{i}}(r)$ with height and thereby must affect a decreased (or increased) internal field strength $\left|B_{\mathrm{i}}(r)\right|$ or an increased (or decreased) external pressure $p_{\mathrm{e}}(r)$ to satisfy the equilibrium Eq. (1). Small changes in internal field strength might be accommodated by changes in the flux-tube size as a state of local stress in the large-scale magnetic field, and changes in the surrounding ambient pressure $p_{\mathrm{e}}(r)$ might be modified by compensating external dynamics like we describe in Sect. 4. Hot flux tubes should tend to cool to the temperature of their immediate surroundings, bringing them back to the normal MHS equilibrium condition. A relatively cool nonevacuated flux tube with excess field strength tends to evacuation with height as a different equilibrium condition, and so might just persist out of equilibrium within about one internal scale height of its base.

Continuous 3D non-force-free equilibrium solutions are claimed (Low 1985, 1991), but these allow an arbitrary hydrostatic 1D gas pressure independent of the magnetic field (see the use of $p_{0}(r)$ and discussion around Eq. (24) of Low 1991, in Sect. III in Bogdan \& Low 1986, or Sect. 4 in Neukirch 1997). In the general force balance, that is the cross-field Eq. (11) with $\phi(r)$ allowed to vary from field line to field line, the pressure and magnetic variations cannot be separated except in force-free regions unconstrained by a horizontal pressure boundary condition, and so necessarily lacking isolated thin non-force-free flux tubes evacuated or in thermal balance with their surroundings.

We have confined our study here to the static solutions, but it is straightforward to broaden consideration to include certain types of steady flows. Steady uniform flows along field lines add a flow pressure to the parallel-field Eq. (5), which leads to a modified hydro-steady relation in Eqs. (9) and (10), but the same cross-field Eq. (11) results. Thus we obtain a gas pressure containing static and wind components coupled to the largescale magnetic field outside flux tubes. For a given gas pressure variation or scale-height function $\phi(r)$ as is defined by the magnetic field in a magnetically dominated environment, the static contribution to the pressure scale height $h(r)$ and corresponding temperature $k T(r)=\bar{m} g(r) h(r)$ are always larger with a steady flow than without (Parker 1960). Steady flux-tube flows can modify the coupling too. 


\section{Model atmospheres}

The derivation of an explicit static equilibrium solution is a formidable task because such an analysis must consider the specific form that distorted magnetic fields take in the presence of a gas. However an approximate coupled scale-height function can be written

$\tilde{\phi}(r)=\phi_{\mathrm{a}}(r)+\frac{1}{\beta_{\mathrm{S}}} \phi_{B 2}(r)$,

where $\phi_{\mathrm{a}}(r)$ and $\phi_{B 2}(r)$ are nominal separate gas and magnetic pressure solutions to the MHS Eqs. (3) and (4) normalized at a base height $r_{\mathrm{s}}$; the coefficient $1 / \beta_{\mathrm{s}}$ defines the relative magnetic-field strength. The approximate coupled scale-height function $\tilde{\phi}(r)$ is proportional to the total pressure $p+\boldsymbol{B}^{2} /(8 \pi)$ of the nominal separate solutions. The gradient of the total pressure approximates the gas-pressure gradient minus the Lorentz term in the MHS equilibrium Eq. (3) reflecting the local balance in Eq. (1), as discussed at the end of Appendix B. The coupled scale-height function $\tilde{\phi}(r)$ thus gives a correct MHS solution where one pressure component is dominant, $p \gg \boldsymbol{B}^{2} /(8 \pi)$ or $\boldsymbol{B}^{2} /(8 \pi) \gg p$.

At a base height $r_{\mathrm{s}}$ taken as the stellar photospheric surface, we assume that magnetic fields are relatively weak, $\beta_{\mathrm{s}} \gg 1$ and $\tilde{\phi}(r) \simeq \phi_{\mathrm{a}}(r)$. There the gas pressure is dominant and must follow its nominal atmospheric form not being much affected by the magnetic field, whereas the magnetic field lines must be highly distorted. The nominal atmospheric-pressure scale-height function $\phi_{\mathrm{a}}(r)$ is defined by the hydrostatic relation Eq. (10) with a temperature found using a transfer equation that includes all of the usual energy input and loss mechanisms.

The atmospheric contribution $\phi_{\mathrm{a}}(r)$ characteristically falls off much more rapidly than the magnetic pressure $\phi_{B 2}(r)$, so around some transition height $r_{\mathrm{t}}$ where $\phi_{\mathrm{a}}\left(r_{\mathrm{t}}\right)=\phi_{B 2}\left(r_{\mathrm{t}}\right) / \beta_{\mathrm{s}}, \tilde{\phi}(r)$ changes from $\phi_{\mathrm{a}}(r)$ to $\phi_{B 2}(r) / \beta_{\mathrm{s}}$ over about one gas-pressure scale height. Above $r_{\mathrm{t}}$ the magnetic field is dominant $\tilde{\phi}(r) \simeq$ $\phi_{B 2}(r) / \beta_{\mathrm{s}}$, and the field must follow its nominal form irrespective of the gas pressure. There the gas pressure profile may be very distorted from its nominal form, representing an added atmospheric heating produced by MHS restoring flows as we discuss in Sect. 4.

Both the magnetic and gas pressure variations must be distorted from their nominal separate forms around the intermediate transition height $r_{\mathrm{t}}$. Preserving the total pressure $p+\boldsymbol{B}^{2} /(8 \pi)$ gives an approximate flux-conserving extrapolation below the magnetically dominated corona and an ostensible gas-pressure extrapolation above the photosphere and chromosphere. The total relative pressure around the intermediate transition height goes from $A_{\text {tot }}\left(\phi_{\mathrm{a}}(r)+\phi_{B 2}(r) / \beta_{\mathrm{s}}\right)$ for a nominal superposition of independent gas and magnetic pressures $\phi_{\mathrm{a}}(r)$ and $\phi_{B 2}(r) / \beta_{\mathrm{s}}$ with $A_{\text {tot }}$ the total surface area, to $\left(A_{\text {tot }}-A_{\text {vac }}\right) \tilde{\phi}(r)+A_{B} \tilde{\phi}(r)$ for the coupled pressure $\tilde{\phi}(r)$ with $A_{\text {vac }}$ the evacuated area and $A_{B}$ the magnetically filled area. The two pressure totals are equal in general only with $\tilde{\phi}(r)$ from Eq. (17) and when the field-filling regions are evacuated with $A_{B}=A_{\mathrm{vac}}$. Anyway the choice of coupled scale-height function is not too critical for our demonstration, as smooth switching functions with correct asymptotic behavior in the photosphere and corona exhibit similar temperature profiles even around the transition height.
For demonstration purposes, we take $\phi_{\mathrm{a}}(r)$ for a polytrope atmosphere as derived in Appendix D

$\phi_{\mathrm{a}}(r)=\left(1-\frac{\Gamma-1}{\Gamma} \frac{r_{\mathrm{s}}}{h_{\mathrm{s}}}\left(1-\frac{r_{\mathrm{s}}}{r}\right)\right)^{\frac{\Gamma}{\Gamma-1}}$,

where $h_{\mathrm{s}} \equiv h\left(r_{\mathrm{s}}\right)=k T_{\mathrm{s}} /\left(\bar{m} g_{\mathrm{s}}\right)$ denotes the surface scale height and $\Gamma$ the ratio of specific heats or polytrope adiabat.

A nominal scale-height function $\phi_{B 2}(r)$ is defined for a single-spherical-harmonic potential magnetic field. The potential magnetic form seems to be consistent with what is observed in the lower solar corona for $r<1.6 r_{\odot}$ (Altschuler \& Newkirk 1969; Schatten et al. 1969). The potential magnetic field vector is the gradient of a sum of scalar spherical-harmonic component functions, each of which is the separable product of a $2 \mathrm{D}$ surface function and radial multiplier $1 / r^{\ell+1}$. The resulting magnetic field in each spherical harmonic goes like $1 / r^{\ell+2}$ in all its vector elements; a monopole $\ell=0$ exhibits a $1 / r^{2}$ radial falloff, a dipole field $\ell=1$, a $1 / r^{3}$ falloff, etc. For a single spherical harmonic $\ell$, the magnetic energy density falls off like

$\phi_{B 2}(r)=\left(\frac{r_{\mathrm{s}}}{r}\right)^{2 \ell+4}$.

With a superposition of spherical harmonics, a nonuniform radial dependence can occur, and a representative globalaverage spherical harmonic $\ell$ might be obtained by appropriately weighting flux-tube locations.

The temperature is written from the hydrostatic Eq. (9)

$k T(r)=\bar{m} g(r) h(r)=-\bar{m} g(r)\left(\frac{1}{\phi(r)} \frac{\partial \phi(r)}{\partial r}\right)^{-1}$.

Taking $\phi(r)$ to be the approximate $\tilde{\phi}(r)$ from Eq. (17) with $\phi_{\mathrm{a}}(r)$ from Eq. (18) and $\phi_{B 2}(r)$ from Eq. (19), we obtain an explicit formula for $T(r)$. In the corona $\phi(r) \simeq \phi_{B 2}(r) / \beta_{\mathrm{s}}$, and the formula exhibits the limiting scale height

$h_{\text {cor }}(r)=\frac{r}{2 \ell+4}$,

and temperature

$k T_{\mathrm{cor}}(r)=\frac{G M_{\mathrm{s}} \bar{m}}{(2 \ell+4) r}=\frac{\bar{m} g(r) r}{2 \ell+4}$.

The limiting base coronal temperature $T_{\mathrm{cor}}\left(r_{\mathrm{s}}\right)$ is defined entirely by the stellar surface gravity $g_{\mathrm{s}}$, radius $r_{\mathrm{s}}$, mean particle mass $\bar{m}$, and main magnetic spherical harmonic $\ell$. For a toroidal field $\ell=2$, the scale height at the stellar surface is $h_{\text {cor }}\left(r_{\mathrm{s}}\right)=r_{\mathrm{s}} / 8$, which gives a solar base coronal temperature of $T_{\text {cor }}\left(r_{\mathrm{s}}\right)=1.73 \times 10^{6} \mathrm{~K}$ (using $\bar{m}=0.6 m_{\mathrm{p}}$ for $m_{\mathrm{p}}$ the proton mass, $\bar{m}=1 \times 10^{-24} \mathrm{~g}, r_{\mathrm{s}}=r_{\odot}=6.96 \times 10^{10} \mathrm{~cm}$, $g_{\mathrm{s}}=2.75 \times 10^{4} \mathrm{~cm} \mathrm{~s}^{-2}$, and $k=1.38 \times 10^{-16} \mathrm{ergs} \mathrm{K}^{-1}$ ). With a solar base coronal temperature of $T_{\text {cor }}\left(r_{\mathrm{s}}\right)=1.6 \times 10^{6} \mathrm{~K}$, we obtain the spherical harmonic $\ell=2.33$.

Figure 2 shows some example scale-height functions $\tilde{\phi}(r)$ from Eqs. (17)-(19) in the lowest part of the solar atmosphere around the transition height $r_{\mathrm{t}}$. Scale-height functions with the polytrope adiabat $\Gamma=1.1$ and spherical harmonic $\ell=2.33$ are plotted for different $\beta_{\mathrm{s}}$, using the solar parameters and surface photospheric temperature $T_{\mathrm{s}}=6820 \mathrm{~K}$ from the VAL model 


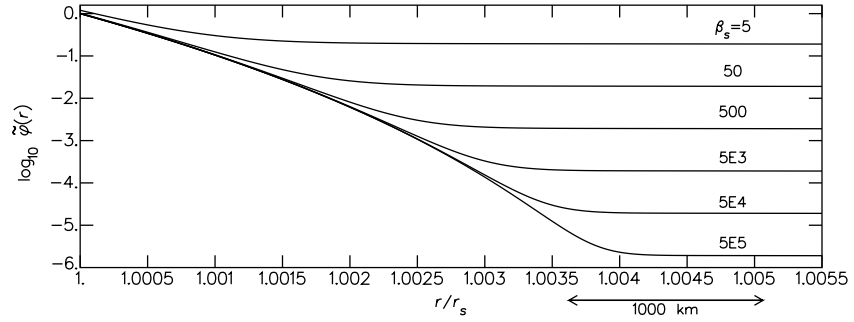

Fig. 2. Log of scale-height function $\log _{10} \tilde{\phi}(r)$ for a solar polytrope atmosphere with $\Gamma=1.1$ and a potential magnetic field $\ell=2.33$ for different $\beta_{\mathrm{s}}$.
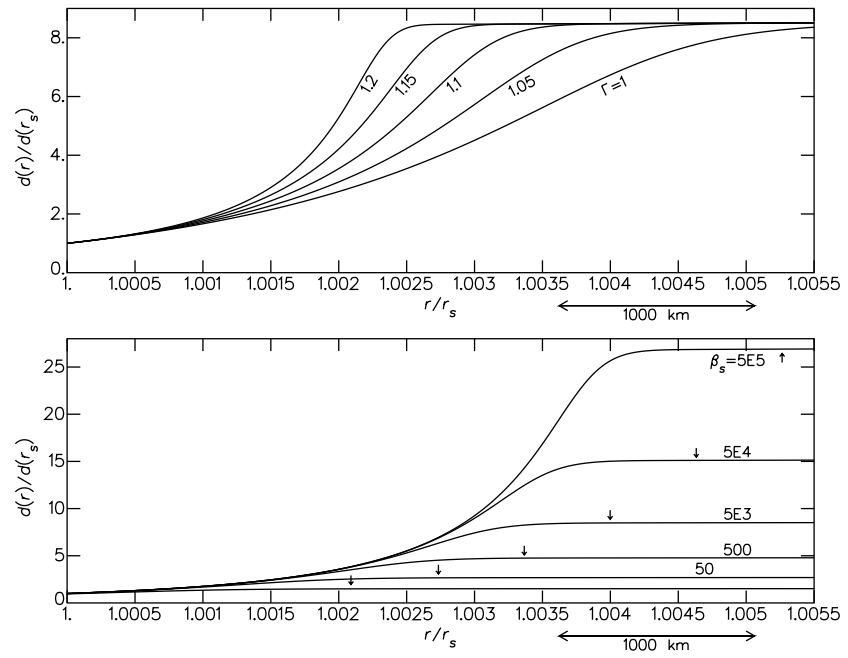

Fig. 3. Relative flux-tube diameter $d(r) / d\left(r_{\mathrm{s}}\right)$ in lower solar atmosphere with $\ell=2.33$ and $\beta_{\mathrm{s}}=5000$ for different polytrope adiabats $\Gamma$ (upper panel), and with $\ell=2.33$ and $\Gamma=1.1$ for different $\beta_{\mathrm{s}}=5$, $50, \ldots 5$.E5 (lower panel). The arrow on each curve locates the most rapid temperature change for the model.

atmospheres (Vernazza et al. 1981), which defines the surface scale height $h_{\mathrm{s}}=k T_{\mathrm{s}} /\left(\bar{m} g_{\mathrm{s}}\right)$ for $\phi_{\mathrm{a}}(r)$ in Eq. (18). As all of the curves are based on the same $\Gamma$, they coincide until the transition height $r_{\mathrm{t}}$ for the model is reached, and then switch rapidly to the nominal magnetic $\phi_{B 2}(r)$, which appears to be relatively flat on the log scale.

The radial expansion of a flux tube is another way to visualize the field strength decrease in the atmosphere and the properties of the coupled scale-height function. For conservation of the total flux through the cross-sectional area of a flux tube $\pi(d / 2)^{2}|\boldsymbol{B}|$, the flux-tube diameter $d(r)$ must increase with radial distance $r, d(r) \propto|\boldsymbol{B}|^{-1 / 2} \propto \phi(r)^{-1 / 4}$. Figure 3 portrays the relative flux-tube diameter as a function of height $d(r) / d\left(r_{\mathrm{s}}\right)$ for different solar atmospheric models denoted by $\Gamma$ and $\beta_{\mathrm{s}}$.

For a given polytrope adiabat $\Gamma$, all flux tubes exhibit approximately the same relative shape up to a height that depends upon $\beta_{\mathrm{s}}$, where the field lines straighten up. Stronger fields exist with a lower transition height and exhibit less overall relative expansion before straightening up. The canopy depends upon the surface distribution of the fields, but strong fields might give the appearance of a lower canopy height too consistent with observations of sunspots (Giovanelli \& Jones 1982). The straightening height is somewhat below the location of the
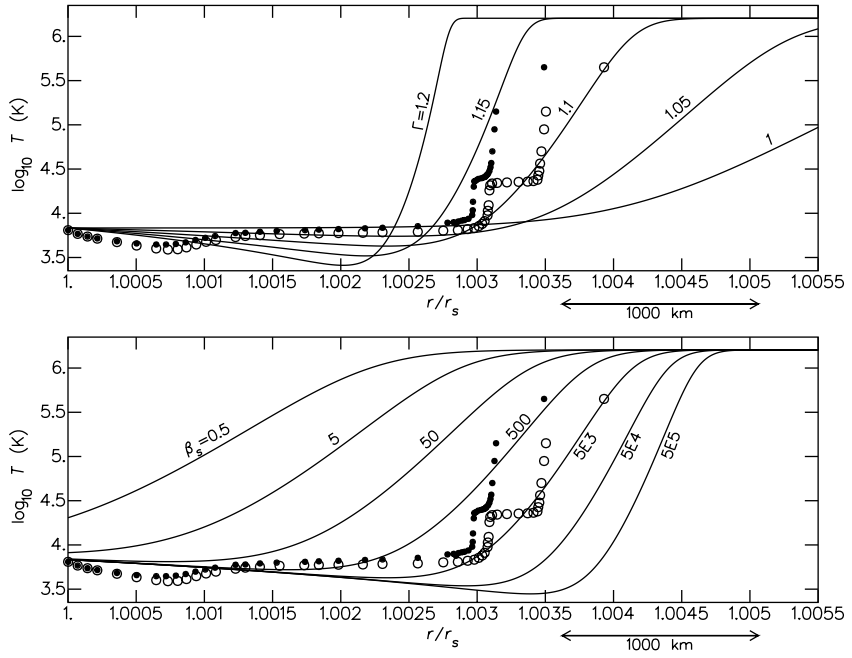

Fig. 4. Lower solar atmosphere $\log _{10} T(r)$ with $\ell=2.33$ and $\beta_{\mathrm{s}}=5000$ for different polytrope adiabats $\Gamma$ (upper panel), and with $\ell=2.33$ and $\Gamma=1.1$ for different $\beta_{\mathrm{s}}$ (lower panel), shown with the solar VAL A (open circles) and $\mathrm{F}$ (filled circles) atmospheres.

fastest temperature change due to the differing dependencies: the field strength, which goes like $\phi(r)^{1 / 2}$, corrects from its atmospherically determined form at $r_{\mathrm{t}}$, but the most rapid temperature change occurs significantly higher where the slope of the scale-height function flattens out, as $T(r) \propto-(\partial \ln \phi(r) / \partial r)^{-1}$.

Figure 4 illustrates the temperature variation in the lower solar atmosphere using Eq. (20) with $T_{\mathrm{s}}=6820 \mathrm{~K}$ from the VAL atmospheres. The upper panel shows the model atmospheres with $\ell=2.33$ and $\beta_{\mathrm{s}}=5000$ for different polytrope adiabats $\Gamma$, and the lower panel the model atmospheres with $\ell=2.33$ and $\Gamma=1.1$ for different $\beta_{\mathrm{s}}$. The VAL $\mathrm{A}$ and $\mathrm{F}$ model temperatures for relatively cool inner network and hot network bright points are also shown.

In the solar photosphere, the nominal atmospheric polytrope follows the VAL temperature roughly, giving the best tradeoff between photospheric and chromospheric temperatures with $\Gamma \simeq 1.1$. Of course the features of a real solar atmosphere can never be well approximated by a polytrope. The shape of the temperature function through the chromosphere and transition height, where the scale-height function $\tilde{\phi}(r)$ goes from $\phi_{\mathrm{a}}(r)$ to $\phi_{B 2}(r) / \beta_{\mathrm{s}}$, depends upon $\Gamma$ and $\beta_{\mathrm{s}}$ but not on $\ell$, which separately determines the base coronal temperature. Temperature profiles with $\beta_{\mathrm{s}} \simeq 5000$ or 1000 give best agreement around the transition height with the VAL A or F atmospheres, respectively, averaging approximately through the Ly $\alpha$ plateau.

For flux-tube evacuation in the photosphere $\boldsymbol{B}\left(r_{\mathrm{s}}\right)^{2}=$ $8 \pi p\left(r_{\mathrm{s}}\right)$, and we obtain a maximum field strength of $\left|\boldsymbol{B}\left(r_{\mathrm{s}}\right)\right|=$ $1715 \mathrm{G}$ using $p\left(r_{\mathrm{s}}\right)=1.17 \times 10^{5} \mathrm{dyn}^{-2}$ from the VAL solar models; $1 / \beta_{\mathrm{s}}^{1 / 2}$ might be taken as the fractional area covered by evacuated magnetic fields. Strictly the coefficient $1 / \beta_{\mathrm{s}}$ represents the coronal base gas or non-force-free magnetic pressure extrapolated back to $r_{\mathrm{s}}$, which can be no larger than the average surface magnetic pressure. Thus the fractional area covered by evacuated magnetic fields at $r_{\mathrm{s}}$ must be at least $1 / \beta_{\mathrm{s}}^{1 / 2}$, or $1 / 71$ for $\beta_{\mathrm{s}}=5000$ and $1 / 32$ for $\beta_{\mathrm{s}}=1000$, which corresponds 


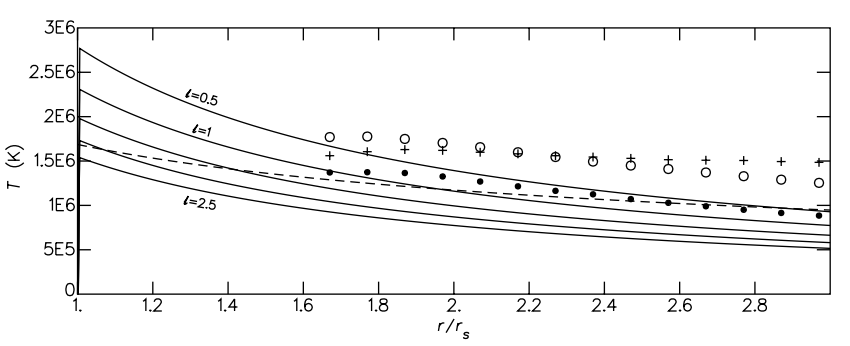

Fig. 5. Static solar corona $T(r)$ for $\ell=.5,1,1.5,2$, and 2.5 (from top to bottom) shown with temperature from white-light intensity in the quiet equator (filled circles), in SW (crosses), and in SE (open circles) streamers (Newkirk et al. 1970), and from line-width measurements (dashed line) (Jarrett \& von Klüber 1958).

to a minimum base coronal field strength of $\left|\boldsymbol{B}_{\text {cor }}\right|=24 \mathrm{G}$ for $\beta_{\mathrm{s}}=5000$ and $\left|\boldsymbol{B}_{\text {cor }}\right|=54 \mathrm{G}$ for $\beta_{\mathrm{s}}=1000$, within the range of solar observations (Lin et al. 2000). A lower transition region occurs where the photospheric flux-tube areal coverage and coronal magnetic field strength are larger near active regions, consistent with the known tendency.

The solar atmospheric model of Fontenla et al. (1990), which includes particle diffusion and conduction effects, lacks the Ly $\alpha$ plateau and exhibits a much more abrupt transition region than what we obtain. While radiative, diffusive, and conductive losses must be largely balanced by MHS restoring flows in the corona, as we discuss in Sect. 4, loss mechanisms can influence the detailed shape of transition-region profiles. Real radiative models coupled to more general magneto-hydrosteady equilibrium solutions need to be developed, but such work may be complicated by the intrinsic limitations and uncertainties in atmospheric modeling, especially associated with inhomogeneous magnetic structure (Ayres 1981; Carlsson \& Stein 1995). It is possible that general methods based upon transition-region emissivity profiles, which have been used to determine the nonradiative atmospheric heating contribution (Craig \& Brown 1976; Anderson et al. 1996), might be able to distinguish coronal heating by MHS restoring flows from other heating mechanisms.

Figure 5 illustrates the solar atmospheric temperature function $T(r)$ for various values of $\ell$ with the coronal temperatures inferred from the white-light-intensity radial gradient taken from eclipse photographs (Newkirk et al. 1970) and inferred from FeXIV 5303 $\mathrm{A}$ line-width measurements (Jarrett \& von Klüber 1958). The limited resolution in $r$ in the figure hides the transition region near $r_{\mathrm{s}}$. The coronal model for the spherical harmonic $\ell=1$ seems to give the best overall agreement with the measured radial profiles, but $\ell \simeq 2.3$ better matches the base coronal temperature. However all of the model curves show a more rapid falloff than the measured temperature profiles. Models containing an outward wind should give systematically higher temperatures with height in the corona.

\section{MHS restoring flows}

Under usual conditions, e.g. quasi-steady, the large-scale and long-term system average is represented by the equilibrium state. In the quasi-steady approximation, the system evolution can be described by a sequence of nearby equilibria. Low (1977) shows that the quasi-steady approximation is applicable when magnetic adjustments are fast compared to evolutionary processes and the magnetic field is dominant over the gas, as in the lower solar corona.

A qualitative feature of MHD processes in a slowly evolving dominant magnetic field where reconnections are fast is an atmospheric restoring flow. Hydro-steady equilibria are always self-restoring. In the absence of sufficient external gas or flow pressure, a time-dependent outward gas acceleration arises outside and along rigidly held non-force-free flux tubes in the overall MHD balance, as described in Appendix E. Flows in a fixed magnetic field act generally to reestablish the equilibrium atmosphere, heating the gas compressively. As long as the timescale for magnetic evolution is relatively long, such MHD processes can have a large-scale effect since the pressure perturbation that drives the flow propagates horizontally away from flux tubes at the sound speed.

The available energy for outflows must be limited and the atmosphere has to eventually cool, distorting and geometrically stressing the coupled magnetic field, which contributes to magnetic instability. The magnetic field can be perturbed in other ways too, for example by new flux coming up from the convection zone below or by photospheric twisting and flux-tube motions. Magnetic evolution is complicated since unstable equilibria are generally possible where perturbations reduce the overall energy of the system. Also local deviations may persist within field allowances before equilibria can be resolved. However if the new magnetic equilibrium after fast reconnection is mostly force-free containing rigidly held non-force-free flux tubes, outflows again must reestablish the equilibrium gaspressure profile of a depleted cool atmosphere, applying the argument from Appendix E.

MHS restoring flows may provide an important coronal heating mechanism, which can exist consistently along with other heating processes. By offseting the total coronal energy requirement, other heating sources should add stability to the large-scale magnetic field and thus longevity to the magnetic evolution. The total coronal energy requirement is estimated conventionally using heat-flux scaling relations for loops and homogeneous regions (Rosner et al. 1978; Hearn \& Kuin 1981; Hammer 1982). If sufficient magnetic energy is not available from evolutionary processes to offset losses, then pure forcefree cool equilibria may be the only possibility.

Localized heating in flux tubes, which is thought to be an important coronal heating process (Vaiana \& Rosner 1978; Airapetian \& Smartt 1995), may affect ambient MHS restoring flows. Heated flux tubes must exhibit field-line perturbations that stress the large-scale magnetic field and tend to produce outflows in the surroundings at least on the average, which heat the ambient atmosphere above the normal equilibrium temperature. The ambient hydrostatic imbalance can have a relatively large scale of influence since it propagates horizontally away from its source much faster than thermal diffusion effects. Such post-reconnection hydrodynamic heating acting throughout the coronal volume may help overcome the often-cited difficulty that reconnection events are so spatially localized and short-lived that only a larger event rate than what is directly 
inferred from observations could account for coronal heating (Heyvaerts \& Priest 1984; Hudson 1991).

Thus MHD processes may lead to refilling outflows, a "magnetic suction", which might be in evidence as the largescale flows seen in the vicinity of coronal voids (Wagner et al. 1983), or be responsible for the supergranular-scale chromospheric upward velocities interpreted from post-flare solar spectral-line blue shifts (Schmieder et al. 1987; Cauzzi et al. 1996), or appear as spicule eruptions in the chromosphere. The upward mass flux produced by solar spicule events is about 100 times the total solar-wind mass flux, but represents only a small fraction of its total energy flux (Pneuman \& Kopp 1978).

The remarkable feature of an MHS corona is that the temperature is essentially a geometric parameter defined by the stellar surface gravity $g_{\mathrm{s}}$, radius $r_{\mathrm{s}}$, mean particle mass $\bar{m}$, and magnetic spherical harmonic $\ell$

$k T_{\text {cor }}\left(r_{\mathrm{s}}\right)=\frac{\bar{m} g_{\mathrm{s}} r_{\mathrm{s}}}{2 \ell+4}$

The observed average solar base coronal temperature of $1.6 \times$ $10^{6} \mathrm{~K}$ corresponds to a spherical harmonic of $\ell=2.33$ consistent with the large-scale solar magnetic field, which exhibits substantial power in the toroidal $\ell=2$ and spherical harmonics $\ell=3$ or 4 as evidenced by the presence of active longitudes. With a general mixture of spherical harmonics, discrepant horizontal gas-pressure boundary conditions at flux tubes can arise, and a nonuniform large-scale surface temperature distribution is possible as a deviation from the pure MHS equilibrium state.

Formulae for the average base coronal temperature like Eq. (23) have been suggested already in the hypothesis of a "geometric boundary condition", relevant for different kinds of heating models where the base pressure scale height is proportional to the stellar radius (Menzel 1968; Scudder 1992). The relation appears to give reasonable coronal temperatures for a range of stellar types (Williams \& Mullan 1996).

Acknowledgements. The author is grateful to many for useful discussion during the course of this work, especially to Eric Priest and Ray Smartt for their careful reviews of the manuscript, and to the referee for many insightful remarks and helpful suggestions.

\section{References}

Airapetian, V. S., \& Smartt, R. N. 1995, ApJ, 445, 489

Altschuler, M. D., \& Newkirk, G. J. 1969, Sol. Phys., 9, 131

Anderson, S. W., Raymond, J. C., \& van Ballegooijen, A. 1996, ApJ, 457, 939

Arendt, U., \& Schindler, K. 1988, A\&A, 204, 229

Ayres, T. R. 1981, ApJ, 244, 1064

Bogdan, T. J., \& Low, B. C. 1986, ApJ, 306, 271

Carlsson, M., \& Stein, R. F. 1995, ApJ, 440, L29

Cauzzi, G., Falchi, A., Falciani, R., \& Smaldone, L. A. 1996, A\&A, 306,625

Cheng, C. Z., \& Choe, G. S. 1998, ApJ, 505, 376

Coles, W. A., \& Harmon, J. K. 1978, J. Geophys. Res., 83, 1413

Craig, I. J. D., \& Brown, J. C. 1976, A\&A, 49, 239
Davis, J. M., Gerassimenko, M., Krieger, A. S., \& Vaiana, G. S. 1975, Sol. Phys., 45, 393

Dungey, J. W. 1953, MNRAS, 113, 180

Dungey, J. W. 1958, Cosmic Electrodynamics (Cambridge University Press)

Fontenla, J. M., Avrett, E. H., \& Loeser, R. 1990, ApJ, 355, 700

Giovanelli, R. G. 1982, Sol. Phys., 80, 21

Giovanelli, R. G., \& Jones, H. P. 1982, Sol. Phys., 79, 267

Guhathakurta, M., Rottman, G. J., Fisher, R. R., Orrall, F. Q., \& Altrock, R. C. 1992, ApJ, 388, 633

Hammer, R. 1982, ApJ, 259, 779

Hearn, A. G., \& Kuin, N. P. M. 1981, A\&A, 98, 248

Heyvaerts, J., \& Priest, E. R. 1984, A\&A, 137, 63

Hudson, H. S. 1991, Sol. Phys., 133, 357

Jarrett, A. H., \& von Klüber, H. 1958, MNRAS, 122, 223

Lin, H., Penn, M. J., \& Tomczyk, S. 2000, ApJ, 541, L83

Lin, J., Zhang, Z. D., Wang, Z., \& Smartt, R. N. 1992, A\&A, 253, 557

Low, B. C. 1975, ApJ, 197, 251

Low, B. C. 1977, ApJ, 217, 988

Low, B. C. 1980, Sol. Phys., 65, 147

Low, B. C. 1985, ApJ, 293, 31

Low, B. C. 1991, ApJ, 370, 427

Low, B. C. 1992, A\&A, 253, 311

MacQueen, R. M., Eddy, J. A., Gosling, J. T., et al. 1974, ApJ, 187, L85

MacQueen, R. M., Sime, D. G., \& Picat, J.-P. 1983, Sol. Phys., 83, 103

Menzel, D. H. 1968, AJ, 73, S71

Neukirch, T. 1997, A\&A, 325, 847

Newkirk, G. J., Dupree, A., \& Schmahl, E. 1970, Sol. Phys., 15, 15

November, L. J., \& Koutchmy, S. 1996, ApJ, 466, 512 (NK96)

Parker, E. N. 1960, ApJ, 132, 821

Parker, E. N. 1972, ApJ, 174, 499

Parker, E. N. 1979, Cosmical Magnetic Fields: Their Origin and Their Activity (Oxford: Clarendon Press)

Parker, E. N. 1987, ApJ, 318, 876

Petschek, H. E., \& Thorne, R. M. 1967, ApJ, 147, 1157

Pneuman, G. W., \& Kopp, R. A. 1978, Sol. Phys., 57, 49

Priest, E. R. 1972, MNRAS, 159, 389

Rosner, R., Tucker, W. H., \& Vaiana, G. S. 1978, ApJ, 220, 643

Schatten, K. H., Wilcox, J. M., \& Ness, N. F. 1969, Sol. Phys., 6, 442

Schmieder, B., Forbes, T. G., Malherbe, J. M., \& Machado, M. E. 1987, ApJ, 317, 956

Scudder, J. D. 1992, ApJ, 398, 299

Syrovatskii, S. I. 1981, ARA\&A, 19, 163

Vaiana, G. S., \& Rosner, R. 1978, ARA\&A, 16, 393

Vaiana, G. S., Zombeck, M., Krieger, A. S., \& Timothy, A. F. 1976, Ap\&SS, 39, 75

Vainshtein, S. I., \& Parker, E. N. 1986, ApJ, 304, 821

van Ballegooijen, A. A. 1985, ApJ, 298, 421

Vernazza, J. E., Avrett, E. H., \& Loeser, R. 1981, ApJS, 45, 635

Wagner, W. J., Newkirk, G. J., \& Schmidt, H. U. 1983, Sol. Phys., 83, 115

Williams, L. L., \& Mullan, D. J. 1996, ApJ, 457, L95

Withbroe, G. L. 1975, Sol. Phys., 45, 301

Woo, R., Armstrong, J. W., Bird, M., \& Patzold, M. 1995, ApJ, 449, L91

Zweibel, E. G., \& Hundhausen, A. J. 1982, Sol. Phys., 76, 261 


\section{Online Material}




\section{Appendix A: Local MHS equilibria}

There are well-known solutions to the classical static problem describing a gas existing in conjunction with elongated magnetic fields without a perturbing gravitational field (Parker 1979, Chap. 6). It is helpful to revisit the classical problem here in a general way. The classical MHS equilibrium equations without gravity are the static equilibrium equation

$\boldsymbol{\nabla} p=\frac{1}{4 \pi}(\boldsymbol{\nabla} \times \boldsymbol{B}) \times \boldsymbol{B}$,

and Gauss' Law, Eq. (4).

Plane-parallel solutions can be developed based upon the general Cartesian vector function

$\boldsymbol{B}=\left(\frac{\partial a(\boldsymbol{x})}{\partial y},-\frac{\partial a(\boldsymbol{x})}{\partial x}-\frac{\partial b(\boldsymbol{x})}{\partial z}, \frac{\partial b(\boldsymbol{x})}{\partial y}\right)$.

The $x$ and $z$ vector elements of $\boldsymbol{B}, \partial a / \partial y$ and $\partial b / \partial y$, are taken to be arbitrary functions, and the $y$ element is written so that Gauss' Law is always satisfied. It is convenient to use derivative forms in the vector elements to avoid complicating integrals in the expression.

Any solutions to Eq. (A.1) must satisfy the two conditions $\boldsymbol{B} \cdot \boldsymbol{\nabla} p=0$ and $(\boldsymbol{\nabla} \times \boldsymbol{B}) \cdot \boldsymbol{\nabla} p=0$, which are written with $\boldsymbol{B}$ from Eq. (A.2)

$\mathrm{C}_{x y} a-\mathrm{C}_{y z} b=0$,

$\left(\mathrm{C}_{x z} \frac{\partial}{\partial x}+\mathrm{C}_{y z} \frac{\partial}{\partial y}\right) a+\left(\mathrm{C}_{x y} \frac{\partial}{\partial y}+\mathrm{C}_{x z} \frac{\partial}{\partial z}\right) b=0$,

using the linear commutation operators $\mathrm{C}_{x y} \equiv \frac{\partial p}{\partial x} \frac{\partial}{\partial y}-\frac{\partial p}{\partial y} \frac{\partial}{\partial x}$, etc., which contain $p(\boldsymbol{x})$ as an implicit function.

The two Eqs. (A.3) and (A.4) can be used to write two of the functions in terms of the third, for example $a(\boldsymbol{x})$ and $b(\boldsymbol{x})$ in terms of $p(\boldsymbol{x})$. The third relation, Eq. (A.1) in the vector direction perpendicular to both $\boldsymbol{B}$ and $\boldsymbol{\nabla} \times \boldsymbol{B}$, gives a differential relation for the third quantity. The separate vanishing of every commutator term is a reduction that is satisfied with certain general functional dependencies between the variables and leads to a well-known self-consistent form for the third relation, the Grad-Shafranov Equation. Other possibilities are not considered here.

Taking every commutator term to vanish in Eqs. (A.3) and (A.4), but without other reduction, gives $a(\boldsymbol{x}), b(\boldsymbol{x})$, and spatial derivatives of $a(\boldsymbol{x})$ and $b(\boldsymbol{x})$ as functions of $p(\boldsymbol{x})$ alone, which is only possible with specific spatial forms for real functions, so other reductions are required in general. Taking $\partial / \partial z=0$ gives a consistent solution defined by the functional dependencies $a=a(p)$ in Eq. (A.3) and $\frac{\partial b}{\partial y}=\frac{\partial b}{\partial y}(p)$ in Eq. (A.4). A similar solution is obtained by taking $\partial / \partial x=0$. These two solutions or linear combinations are equivalent with rotation of the $x-z$ axes around $y$. Taking $z$ as the degenerate direction gives the usual 2D solution, ordinarily written with $a$ as the implicit function

$\boldsymbol{B}=\left(\frac{\partial a(x, y)}{\partial y},-\frac{\partial a(x, y)}{\partial x}, B_{z}(a(x, y))\right)$.

The degenerate $z$ is a direction of field elongation. The magnetic potential function $a(x, y)$ defines a $2 \mathrm{D}$ planform for the solutions, which determines the spatial variations in the longitudinal field element $B_{z}=B_{z}(a(x, y))$ and gas pressure $p=p(a(x, y))$ as arbitrary 1D mappings. The magnetic field is everywhere perpendicular to the gradient of $a(x, y)$, since $\boldsymbol{B} \cdot \boldsymbol{\nabla} a(x, y)=0$.

It is widely believed that this $2 \mathrm{D}$ solution is the only planeparallel one. Using perturbative expansions, Parker (1979, Sect. 14.2) shows that no nearby 3D solutions exist for bounded quantities in an infinite spatial domain. However 3D variations do arise as large-scale deviations from plane parallel (Arendt \& Schindler 1988). In the common shorthand, "2.5D" solutions refer to perturbed solutions in the problem with gravity, which admit large-scale variations in the degenerate direction of the magnetic field.

\section{Appendix B: Properties of local MHS equilibria}

Substituting $\boldsymbol{B}$ from Eq. (A.5) into Eq. (A.1) gives the governing equation for the implicit magnetic potential function $a(x, y)$ for the static equilibrium problem without gravity

$\left(\nabla^{2} a+\frac{\mathrm{d}}{\mathrm{d} a}\left(4 \pi p(a)+\frac{B_{z}(a)^{2}}{2}\right)\right) \nabla a=0$.

Where $\nabla a \neq 0$, the potential function must satisfy the GradShafranov (GS) equation, written

$\nabla^{2} a(x, y)=-P^{\prime}(a(x, y))$,

defining the total pressure $P(a)=4 \pi p(a)+B_{z}(a)^{2} / 2$, where $P^{\prime}(a)=\mathrm{d} P(a) / \mathrm{d} a$.

The total pressure $P(a)$ and its component functions $p(a)$ and $B_{z}(a)$ are all one dimensional and nonlinear, but must all be well-defined everywhere in the solution domain consistent with their forms at the boundaries. Strictly, disagreeing $z$ boundary conditions are inconsistent with the $z$ independence of the solutions; differences might produce small deviations in the solutions like twist or divergence or be a source of dynamical instability. It is popular to restrict consideration to entirely force-free solutions with $p^{\prime}(a)=0$; the restriction does not change the nature of the basic GS Eq. (B.2) for $a(x, y)$ but requires the specific current density $\boldsymbol{J}=\frac{c}{4 \pi} B_{z}^{\prime}(a) \boldsymbol{B}$ for an arbitrary $B_{z}(a)$, as is evident by expanding $\boldsymbol{J} \equiv \frac{c}{4 \pi} \boldsymbol{\nabla} \times \boldsymbol{B}$ using $\boldsymbol{B}$ from Eq. (A.5) with $\boldsymbol{J} \propto \boldsymbol{B}$.

The magnetic potential function $a$ can be seen to be constant everywhere in the local plane of $\boldsymbol{B}$ and $\boldsymbol{J}$. From Eq. (A.1), $\boldsymbol{J} \cdot \boldsymbol{\nabla} p(a)=0$. Then $\boldsymbol{J} \cdot \boldsymbol{\nabla} p(a)=(\boldsymbol{J} \cdot \boldsymbol{\nabla} a) p^{\prime}(a)=0$. Thus $\boldsymbol{J} \cdot \boldsymbol{\nabla} a=0$ at least when $p^{\prime}(a) \neq 0$, and when $p^{\prime}(a)=0, \boldsymbol{J} \| \boldsymbol{B}$ so $\boldsymbol{J} \cdot \boldsymbol{\nabla} a=0$ anyway. We think of $a$ as constant on ribbon-like current sheets, the solution surfaces being more curved along $\boldsymbol{J}$ as it is defined by the derivatives of $\boldsymbol{B}$.

The GS Eq. (B.2) has the unusual feature that it contains the 1D filter function $P^{\prime}(a)$. The Fourier transform of the GS equation in the linear case, with $P^{\prime}(a)=k_{0}^{2} a$ for $k_{0}$ a constant wavenumber, requires a transform function $\bar{a}\left(k_{x}, k_{y}\right)$ that is zero everywhere in its 2D wavenumber domain $\boldsymbol{k}=\left(k_{x}, k_{y}\right)$ except on a thin annulus at $|\boldsymbol{k}|=k_{0}$, where arbitrary complex values Hermitian in $\pm \boldsymbol{k}$ are allowed. Taking the Fourier transform back gives the potential function $a(x, y)$ as a common Besselfunction radial kernel times azimuthal factor convolved with a 


\section{J. November: MHS atmospheres, Online Material p 3}

spatial distribution of delta-function source points. Boundary conditions on $a(x, y)$ constrain the azimuthal factor, leading to possible planar solutions defined by all power at one azimuth, or axisymmetric solutions with power uniformly distributed in azimuth, consistent with our visualization of current sheets and cylindrical flux tubes. Physical arguments show that nonlinear kernels are similarly constrained by boundary conditions (Vainshtein \& Parker 1986).

Random spatial distributions of a common GS kernel are nonlinear GS solutions too. We take the potential function $a(x, y)$ to be the convolution of a distribution of sources $D(x, y)$ with a common 2D kernel function $A(x, y), a(x, y)=D(x, y) *$ $A(x, y)$. The distribution is written $D(x, y)=\sum_{j} c_{j} \delta\left(x-x_{j}, y-\right.$ $y_{j}$ ) counting sources $j$ of varying strength $c_{j}$, where $\delta(x, y)$ denotes the 2D delta function. For a spatially incoherent or random distribution $D$ of equal strength sources with all $c_{j}=1$, $D$ raised to a power is $D$ alone; for equal amplitude sources with $c_{j}= \pm 1, D$ raised to an odd power is $D$ alone. For an analytic nonlinear driver function $P(a)$ the convolution factors out $P^{\prime}(a)=P^{\prime}(D * A)=D * P^{\prime}(A)$, when the distribution in $D$ is incoherent, disjoint, and contains suitably restricted amplitudes. Then the GS Eq. (B.2) reduces to the same GS equation, but for the common kernel function $A$ in place of $a$. Dark and bright threads appear to be isolated thin flux tubes aligned with the background magnetic field organized in current sheets suggestive of a bimodal amplitude distribution of a common flux-tube kernel.

Parker (1972) considers MHS equilibria that contain a relatively strong constant background field. Expanding the Lorentz term in Eq. (A.1) gives an alternate form for the MHS equation

$\boldsymbol{\nabla}\left(p+\frac{\boldsymbol{B}^{2}}{8 \pi}\right)=\frac{1}{4 \pi}(\boldsymbol{B} \cdot \boldsymbol{\nabla}) \boldsymbol{B}$,

or with the superposition $\boldsymbol{B}(\boldsymbol{x})=\boldsymbol{B}_{\mathrm{e}}+\boldsymbol{B}_{\sim}(\boldsymbol{x})$ for a constant background field $\boldsymbol{B}_{\mathrm{e}}$ and a spatially varying field $\boldsymbol{B}_{\sim}(\boldsymbol{x})$, we obtain

$\boldsymbol{\nabla}\left(p+\frac{\boldsymbol{B}^{2}}{8 \pi}\right)=\frac{1}{4 \pi}\left(\boldsymbol{B}_{\mathrm{e}} \cdot \boldsymbol{\nabla}\right) \boldsymbol{B}_{\sim}+\frac{1}{4 \pi}\left(\boldsymbol{B}_{\sim} \cdot \boldsymbol{\nabla}\right) \boldsymbol{B}_{\sim}$.

Taking the divergence, applying Gauss's Law $\boldsymbol{\nabla} \cdot \boldsymbol{B}_{\sim}=0$, and using the property that the total pressure $p+\boldsymbol{B}^{2} /(8 \pi)$ is a bounded quantity in an infinite domain, we obtain

$\left(\boldsymbol{B}_{\mathrm{e}} \cdot \boldsymbol{\nabla}\right) \boldsymbol{B}_{\sim}=0$,

at least to first order in the small quantity $\left|\boldsymbol{B}_{\sim}\right| /\left|\boldsymbol{B}_{\mathrm{e}}\right|$. Thus we have

$\boldsymbol{\nabla}\left(p+\frac{\boldsymbol{B}^{2}}{8 \pi}\right) \simeq 0$.

with equality to first order in $\left|\boldsymbol{B}_{\sim}\right| /\left|\boldsymbol{B}_{\mathrm{e}}\right|$. Equation (B.5) proves to be accurate to all orders in the perturbative expansions, and the equilibrium Eqs. (1) and (2) are justified at least in the presence of a relatively strong constant background field, where the internal flux-tube field is defined $\boldsymbol{B}_{\mathrm{i}} \equiv \boldsymbol{B}_{\mathrm{e}}+\boldsymbol{B}_{\sim}(=\boldsymbol{B})$. Additional discussion on Parker's perturbative expansion is contained in Appendix C.
The Parker theorem Eq. (B.5) reflects the special features of the classical equilibria in Eq. (A.5) in the degenerate direction of the magnetic field $z$. MHS equilibria allow only certain alignments for the background field. A constant magnetic field can enter into the $z$ vector element $B_{z}(a)$ without producing other ramifications, whereas an added offset in the $x$ or $y$ elements of $\boldsymbol{B}$ requires adding a uniformly inclined plane to $a(x, y)$. Such a plane limits the solutions and thus cannot be considered general, and even appears to be precluded by the boundedness of $a(x, y)$ : low-wavenumber components in the Fourier domain needed to represent the added plane are at odds with the Fourier ring solutions in the linear case of the GS Eq. (B.2), and also contrary to the form of a superposition of spatially compact axisymmetric nonlinear kernels.

\section{Appendix C: Perturbative expansions}

The less-significant order $n>0$ equations in the perturbative series developed from Eqs. (13) and (14) are written for $\zeta(r)=$ 1; from the MHS Eq. (13)

$$
\begin{array}{r}
\boldsymbol{\nabla} \hat{p}_{1}-\frac{1}{4 \pi}\left(\left(\boldsymbol{\nabla} \times \hat{\boldsymbol{B}}_{0}\right) \times \hat{\boldsymbol{B}}_{1}+\left(\boldsymbol{\nabla} \times \hat{\boldsymbol{B}}_{1}\right) \times \hat{\boldsymbol{B}}_{0}\right)= \\
\frac{1}{8 \pi d}\left(\boldsymbol{\nabla} r \times \hat{\boldsymbol{B}}_{0}\right) \times \hat{\boldsymbol{B}}_{0},
\end{array}
$$

or for the general order $n>0$

$$
\begin{aligned}
& \boldsymbol{\nabla} \hat{p}_{n}- \frac{1}{4 \pi} \sum_{j=0}^{n}\left(\boldsymbol{\nabla} \times \hat{\boldsymbol{B}}_{j}\right) \times \hat{\boldsymbol{B}}_{n-j}= \\
& \frac{1}{8 \pi d} \sum_{j=0}^{n-1}\left(\boldsymbol{\nabla} r \times \hat{\boldsymbol{B}}_{j}\right) \times \hat{\boldsymbol{B}}_{n-j-1}\left(1+2 j \frac{\partial h(r)}{\partial r}\right) .
\end{aligned}
$$

From Eq. (14)

$\boldsymbol{\nabla} \cdot \hat{\boldsymbol{B}}_{1}=-\frac{1}{2 d}\left(\boldsymbol{\nabla} r \cdot \hat{\boldsymbol{B}}_{0}\right)$,

or in general for $n>0$

$\boldsymbol{\nabla} \cdot \hat{\boldsymbol{B}}_{n}=-\frac{1}{2 d}\left(\boldsymbol{\nabla} r \cdot \hat{\boldsymbol{B}}_{n-1}\right)\left(1+2(n-1) \frac{\partial h(r)}{\partial r}\right)$.

The equations are linear in the variables $\hat{p}_{n}$ and $\hat{\boldsymbol{B}}_{n}$ based upon order $<n$ variables. The order-zero base functions $\hat{p}_{0}$ and $\hat{\boldsymbol{B}}_{0}$ are $2 \mathrm{D}$ and constant in the degenerate magnetic field direction $z$; $3 \mathrm{D}$ effects can be introduced with the cross-product terms or with general variation in the function $h(r)$ with $r$ not perpendicular to $z$. For consistency in Eqs. (13) and (14), $\hat{\boldsymbol{B}}_{0} \cdot \boldsymbol{\nabla} \hat{p}_{0}=0$, which is the order-zero component of $\boldsymbol{B} \cdot \boldsymbol{\nabla} \hat{p}=0$.

A wide latitude is allowed for choosing perturbative quantities, and as long as they are relatively small almost everywhere the standard procedure remains valid. Van Ballegooijen (1985, Appendix A) questions the Parker (1972) choice of perturbative quantity and equation separation procedure, but van Ballegooijen's assigning of approximate magnitudes to individual terms to separate his equations is not an algebraic procedure, and his resulting equations have inherent contradictions as described by Parker (1987). Parker (1972) does not ascribe a particular functional meaning to his kernel $\epsilon$, quoting from the beginning of his Sect. II: "Expand the field $\boldsymbol{b}$ and pressure $p$ in 


\section{J. November: MHS atmospheres, Online Material $p 4$}

ascending powers of some parameter $\epsilon$, which is of the order of $\epsilon=|\boldsymbol{b}| /|\boldsymbol{B}|$ ", where $\boldsymbol{b}$ is the small spatially varying magnetic field on the uniform background field $\boldsymbol{B}$. Our choice for perturbative kernel $d / h(r)$ with $d$ constant leads to a convenient separation of orders.

The perturbative procedure is a functional separation method rooted in the natural independence of the basis functions $\epsilon^{n}$ irrespective of their real amplitudes. If the parameter $\epsilon$ is small almost everywhere, the series solutions will be convergent. There could be locations where the amplitudes of components seem to be of differing orders as van Ballegooijen contends. Where a normally small expansion parameter $\epsilon$ actually becomes relatively large series expansions may be divergent, but such behavior characteristically defines the isolated singularities of differential equations as discussed in theorems on the Frobinius method for series solutions.

\section{Appendix D: Polytrope atmosphere}

A polytrope atmosphere is defined by the pressure relation $p(r) \propto \rho(r)^{\Gamma}$ for the polytrope adiabat $\Gamma$. Thus the 1D polytrope scale height $h(r)=p(r) /(\rho(r) g(r))$ can be written

$\frac{h(r)}{h_{\mathrm{s}}}=\left(\frac{p(r)}{p\left(r_{\mathrm{s}}\right)}\right)^{\frac{\Gamma-1}{\Gamma}} \frac{g_{\mathrm{s}}}{g(r)}=\phi(r)^{\frac{\Gamma-1}{\Gamma}} \frac{g_{\mathrm{s}}}{g(r)}$,

making reference to a base height $r_{\mathrm{s}}$ taken to be the stellar surface, and introducing the scale-height function $\phi(r)$ for the normalized gas pressure.

Substituting $h(r)$ into the hydrostatic Eq. (9) defines the scale-height function for a polytrope

$\phi(r)^{-1 / \Gamma} \frac{\partial \phi(r)}{\partial r}=-\frac{g(r)}{g_{\mathrm{s}} h_{\mathrm{s}}}$,

or

$\frac{\partial}{\partial r} \phi(r)^{\frac{\Gamma-1}{\Gamma}}=-\frac{\Gamma-1}{\Gamma} \frac{g(r)}{g_{\mathrm{s}} h_{\mathrm{s}}}$,

giving

$\phi(r)=\left[-\frac{\Gamma-1}{\Gamma} \int \frac{g(r)}{g_{\mathrm{s}} h_{\mathrm{s}}} \mathrm{d} r\right]^{\frac{\Gamma}{\Gamma-1}}$,

and with $g(r)=g_{\mathrm{s}}\left(r_{\mathrm{s}} / r\right)^{2}$, we obtain

$\phi(r)=\left[1-\frac{\Gamma-1}{\Gamma} \frac{r_{\mathrm{s}}}{h_{\mathrm{s}}}\left(1-\frac{r_{\mathrm{s}}}{r}\right)\right]^{\frac{\Gamma}{\Gamma-1}}$,

choosing the constant of integration so that $\phi\left(r_{\mathrm{s}}\right)=1$. The part of the expression contained in brackets [ ] corresponds to the radial temperature variation, the thermal lapse rate. The polytrope adiabat $\Gamma$ ranges from 1 to $5 / 3$ for an atmosphere ranging from lossy and isothermal to adiabatically stratified. For $\Gamma=1$, the scale-height function $\phi(r)$ has a limiting pure exponential decay form. For $\Gamma>1$, the thermal lapse rate and scale-height function have the unphysical feature that they become negative at some height, showing that a polytrope of constant $\Gamma$ can exist only over a limited range in $r$. Such behavior is well known in stellar-interior polytrope models and leads to convection zones of finite extent.

\section{Appendix E: Hydrodynamics around fixed fields}

If the magnetic field is dominant over the gas, and either the field remains stationary, or the magnetic adjustment time is much shorter than the hydrodynamic adjustment time, then the Lorentz term in the MHD equation can be treated as constant in time and the hydrodynamics separate from the magnetodynamics.

Fast magnetic adjustment is a remarkable feature of solar reconnection phenomena. Sporadic crossings of coronal loops in low-energy flare events (Lin et al. 1992) suggest "X"-type configurations, and the instability of the " $\mathrm{X}$ " configuration is well known to form a new current sheet at near the Alfvén velocity if a nonzero resistivity can be affected in the region (Dungey 1958; Syrovatskii 1981); such a resistivity is predicted in some models (Petschek \& Thorne 1967; Priest 1972). Taking a field strength of $|\boldsymbol{B}| \simeq 400 \mathrm{G}$ as representative of chromospheric magnetic fields near neutral lines in solar active regions where flares commonly occur, we obtain an Alfvén speed $c_{\mathrm{a}}=|\boldsymbol{B}| /(4 \pi \rho)^{1 / 2} \simeq 10^{8} \mathrm{~cm} \mathrm{~s}^{-1}$ (with $\rho=N \bar{m}=10^{-12} \mathrm{~g} \mathrm{~cm}^{-3}$, using $N=10^{12} \mathrm{~cm}^{-3}$ and $\left.\bar{m}=10^{-24} \mathrm{~g}\right)$. The Alfvén speed stays fairly constant into the corona giving a collapse time of $\tau_{B}=$ $h_{B} / c_{\mathrm{a}} \simeq 160 \mathrm{~s}$ for the propagation of the instability through a magnetic scale height $h_{B}=2 h_{\text {cor }}=r_{\mathrm{s}} /(\ell+2) \simeq 1.6 \times 10^{10} \mathrm{~cm}$, using a primary magnetic spherical harmonic of $\ell=2.33$ in Eq. (21).

The hydrodynamic response to already present or newly formed isolated thin non-force-free flux tubes can be obtained by perturbative analysis of the dynamical equations projected along the magnetic field. Taking the velocity to be aligned with the magnetic field and of lesser order than the mean quantities adds a single time-derivative term to the projected MHS Eq. (3), which is written with the continuity equation

$\rho \frac{\partial u_{1}}{\partial t}+\frac{\partial p}{\partial z}+\rho g(\nabla z \cdot \nabla r)=0$,

$\frac{\partial \rho}{\partial t}+\frac{\partial}{\partial z}\left(\rho u_{1}\right)=0$

the equations are taken with the thermodynamic energy equation $p \propto \rho^{\gamma} ; z$ is the direction of the magnetic field; $u_{1}$ denotes the velocity amplitude; $\gamma$ is the ratio of specific heats, which ranges from 1 to $5 / 3$ for lossy isothermal refilling flows to adiabatic. We now choose to use the density $\rho$ as a problem variable, rather than the temperature or scale height $h$.

Perturbing the quantities backward in time, $p=p_{0}-p_{1}$ and $\rho=\rho_{0}-\rho_{1}$, from a final equilibrium state $\left(p_{0}, \rho_{0}\right)$ leaves the hydrostatic relation Eq. (9) with $p_{0}$ coupled to the magnetic field through the cross-field Eq. (11), and three coupled perturbative equations for $\left(p_{1}, \rho_{1}, u_{1}\right)$

$$
\begin{aligned}
& \rho_{0} \frac{\partial u_{1}}{\partial t}=\frac{\partial p_{1}}{\partial z}+\rho_{1} g \cos \theta \\
& \frac{\partial \rho_{1}}{\partial t}=\frac{\partial}{\partial z}\left(\rho_{0} u_{1}\right),
\end{aligned}
$$

and $p_{1}=\gamma\left(p_{0} / \rho_{0}\right) \rho_{1}$, introducing $\theta$ as the angle between the magnetic field direction $z$ and the radial $r$. With small positive pressure and density deficits $p_{1}$ and $\rho_{1}$, a small positive velocity $u_{1}$ is required in Eq. (E.3) corresponding to an upward 
flow opposite the normal gravitational stratification. Refilling changes the gas-pressure profile, in effect heating the gas compressively as the dominant heating term in the thermodynamic energy equation.

The unperturbed density $\rho_{0}=p_{0} /(g h)$ varies on a scale similar to the gas pressure $p_{0}$, since the gravity $g$ and temperature contained in $h$ vary on much larger scales; thus the derivative is approximated $\partial \rho_{0} / \partial z \rightarrow \rho_{0} \cos \theta / h$. The perturbed quantities must vary on the scale of the magnetic field $2 h / \cos \theta$, giving $\partial\left(p_{1}, u_{1}\right) / \partial z \rightarrow\left(p_{1}, u_{1}\right) \cos \theta /(2 h)$. Substituting for the time derivative $\partial / \partial t \rightarrow 1 / \tau_{u}$ lets us solve for the hydrodynamic timescale

$$
\tau_{u}=\frac{1}{\cos \theta}\left(\frac{4 h}{(6+3 \gamma) g}\right)^{1 / 2},
$$

which is proportional to the gravitational free-fall time through the scale height $h$. For a radial field $\theta=0$, mixed lossy conditions $\gamma=1.2$, and $h=r_{\mathrm{s}} /(2 \ell+4)$, we obtain $\tau_{u}=349 \mathrm{~s}$ for solar surface conditions with $\ell=2.33$, which is just over twice the magnetic adjustment time $\tau_{B}$. The hydrodynamic adjustment time increases with more lossy conditions and smaller $\gamma$, but overall depends only weakly on the thermodynamics. 